\title{
Free Clinic Diagnosis Data Improvement Project Using International Classification of Diseases and Electronic Health Record
}

\author{
Sarah M. Shanahan, MSN, RN
}

Objective: This quality improvement project aimed to enhance The Olympia Free Clinic's (TOFC) data availability using International Classification of Diseases (ICD) code entry into the electronic health record (EHR). Prior to this project, TOFC lacked quality diagnosis data. This project strived to answer questions like "How many TOFC patients have diabetes?"

Methods: A new system was implemented for inputting $I C D$ codes into Practice Fusion, the clinic's EHR. During the initial phase, TOFC's 21 volunteer providers entered the codes associated with the appropriate diagnosis for each of 157 encounters using a simplified map of options, including a map of the 20 most common diagnoses and a more comprehensive 60-code map.

Results: An EHR report found that 128 new diagnoses were entered during project implementation, hypertension being the most common diagnosis, followed by depression, then posttraumatic stress disorder.

Conclusion: The knowledge of patient diagnoses enabled the clinic to make more-informed decisions.

Keywords: free clinic, data, quality improvement, electronic health record, International Classification of Diseases

( ata creates a starting point, a goal, background, understanding of needs and context, and allows for tracking and improvement over time. This quality improvement (QI) project for The Olympia Free Clinic (TOFC) implemented a new system for tracking patient diagnoses. The 21 primary TOFC providers were encouraged to input mapped International Statistical Classification of Diseases and Related Health Problems (ICD) codes into the electronic health record (EHR). The clinic's providers consisted of mostly retired, but some actively practicing, medical doctors, doctors of osteopathy, nurse practitioners, physician assistants, and psychiatrists.
Previous to this project, the clinic lacked any concrete data on patient demographics or diagnoses. For example, the clinic was unable to accurately answer the National Association of Free and Charitable Clinics' questions about how many patients TOFC providers saw with diabetes, hypertension, asthma, and hyperlipidemia. ${ }^{1}$ Additionally, the needs of the clinic and its population were based on educated guesses.

As a free clinic staffed by volunteers and open 2 days a week, TOFC focused solely on giving care to those who needed it, operating pragmatically and addressing any issues as they arose. However, this strategy left the clinic unable to answer questions like "How many TOFC patients have diabetes?" By answering these questions, the clinic can better assess their resource and staffing needs.

\section{Purpose}

The project enlisted 21 volunteer providers to record diagnoses through ICD codes on the approximately 2000 active patients between March 22, 2021, and June 15, 2021. Tracking patient diagnoses improves clinic data, outcomes, and decision-making. By working on data improvement, the clinic can better understand its patient population and their needs, enhance clinical care, create better outcomes, make informed decisions, and raise eligibility for grants. The clinic was at a turning point as they reevaluated their mission statement and decided whether they would continue to focus on acute ailments or expand to formally manage chronic diseases as well. This decision needed to be made with knowledge, understanding, and context, which diagnosis data can provide. For example, the knowledge that the clinic's 3 most common diagnoses are chronic conditions

From Pacific Lutheran School of Nursing, Tacoma, WA. 
demonstrated that an official shift in their mission may have been warranted.

\section{Literature Review}

QI projects are effective and common in the free clinic setting. ${ }^{2-4}$ To the author's knowledge, no literature to date shows the implementation of a system to better track diagnoses using a free clinic's EHR with ICD codes.

Data bring value to clinics in many ways. It can also lead to more informed and better distribution of resources, such as preventative health and social services, patient education, and medical inventory. ${ }^{4}$

The focus of the US health care system is shifting to a value-based system under the Patient Protection and Affordable Care Act. ${ }^{5}$ Outcome measurements and improvement play a key role in this. ${ }^{6}$ Without knowing diagnoses, we cannot effectively track outcomes and have no data on which to base improvements. Insurance and reimbursement requirements typically hold health care facilities accountable for making these outcomes and improvements a reality. ${ }^{5,6}$ Free clinics, however, lack these motivations, which explains why a free clinic may be deficient in data and tracking methods. Tracking diagnosis codes will, going forward, allow TOFC to see outcomes and trends over time, track the effectiveness of the treatments, and change course if need be. ${ }^{6}$

TOFC fully implemented the EHR in 2018, giving the clinic better capabilities for pulling reports and tracking data. Although there were growing pains, many TOFC providers were already familiar with $I C D$ codes, which, along with an EHR, provide a system to easily retrieve, store, and analyze diagnoses for evidence-based and informed decision-making. This made using ICD codes and the EHR an obvious choice to track patient diagnoses. However, most of the providers were not putting them in $I C D$ codes before this project was implemented. Instead, diagnoses were typed in the notes and, therefore, not easy to generate in a report without having to open each chart for each individual encounter and combing through the notes. To make matters worse, providers were never trained on how to enter the codes in the EHR, and most providers saw no reason to, because the clinic does not bill for services.

\section{Methods}

A needs assessment determined that TOFC lacked data. This QI project used a combination of primary and secondary continuous quality improvement data. ${ }^{8}$ The primary data came from pulling the reports on Practice Fusion to see how many times each diagnosis code was put in during the implementation phase of this project. Secondary data came from interviewing the providers and asking whether they put in the diagnosis codes.

\section{ICD diagnosis entry}

Practice Fusion is the EHR TOFC uses and was therefore the platform for this QI project. Two ICD maps were created, which incorporated both International Classification of Diseases, Ninth Revision (ICD-9) and International Classification of Diseases, Tenth Revision (ICD-10) codes. There are tens of thousands of ICD codes in existence, but because TOFC is a free clinic that does not bill or receive reimbursement, the codes did not need to be as specific as they do in a paid clinic. Therefore, the maps put all the variations of each disease into a single category. For example, every patient with diabetes would receive the same ICD code regardless of whether their diabetes was controlled, uncontrolled, or any other variation. The goal of simplifying the codes was to improve compliance with $I C D$ code entry and make reports easier to generate. The maps allowed the options to be simplified and, therefore, more user friendly for both the providers and the data collectors pulling reports. As some ICD-9 codes were already being used, these codes were incorporated so providers could keep using what they were already familiar with. To create the map, generic $I C D$ codes were selected to represent each disease.

An initial survey was conducted prior to implementation with 10 providers, 2 nurses, and 2 staff members, asking which diagnoses they thought were seen most often in the clinic. Based off those answers, a map was created with the 20 most commonly used ICD codes, which can be seen in the Table. A more comprehensive map was also created, with 61 encompassing diagnoses.

To start the implementation process, providers were emailed an explanation of the project, the ICD code maps, and step-by-step instructions on how to enter a diagnosis 
Table. 20 Most Common Diagnoses With Results ${ }^{a}$

\begin{tabular}{|c|c|c|c|c|}
\hline Diagnosis & ICD Code & Preimplementation $^{\mathrm{b}}$ & Implementation $^{c}$ & Increase \\
\hline Abdominal pain & R10.9 Unspecified abdominal pain & 3 & 6 & 3 \\
\hline Anxiety & 300.00 Anxiety state, unspecified & 113 & 121 & 8 \\
\hline Asthma & 493.90 Asthma, unspecified type & 78 & 79 & 1 \\
\hline Back pain & M54.9 Dorsalgia, unspecified & 6 & 7 & 1 \\
\hline Contraception & $\begin{array}{l}\text { Z30.09 Encounter for other general } \\
\text { counseling and advice on contraception }\end{array}$ & 0 & 1 & 1 \\
\hline COPD & 496 Chronic airway obstruction & 32 & 38 & 6 \\
\hline Depression & $\begin{array}{l}311 \text { Depressive disorders not } \\
\text { elsewhere classified }\end{array}$ & 126 & 151 & 25 \\
\hline Diabetes & $\begin{array}{l}\text { E11.9 Type } 2 \text { diabetes mellitus without } \\
\text { complications }\end{array}$ & 81 & 86 & 5 \\
\hline Headache & R51 Headache & 4 & 7 & 3 \\
\hline Hyperlipidemia & $\begin{array}{l}272.4 \text { Other and unspecified } \\
\text { hyperlipidemia }\end{array}$ & 6 & 10 & 4 \\
\hline Hypertension & 401.9 Unspecified essential hypertension & 136 & 171 & 35 \\
\hline Infertility & N97.9 Female infertility, unspecified & 0 & 0 & 0 \\
\hline Joint pain & M25.50 Pain in unspecified joint & 3 & 4 & 1 \\
\hline $\begin{array}{l}\text { Mood disorder (other than } \\
\text { depression) }\end{array}$ & $\begin{array}{l}296.90 \text { Unspecified episodic mood } \\
\text { disorder }\end{array}$ & 1 & 3 & 2 \\
\hline PTSD & F43.1 Posttraumatic stress disorder & 60 & 83 & 23 \\
\hline $\begin{array}{l}\text { Skin issues (including } \\
\text { cellulitis and rash) }\end{array}$ & $\begin{array}{l}\text { L98.9 Disorder of the skin and } \\
\text { subcutaneous tissue, unspecified }\end{array}$ & 0 & 3 & 3 \\
\hline STI & Z11.3 Encounter for STI screening & 16 & 18 & 2 \\
\hline Upper respiratory infection & $\begin{array}{l}465.9 \text { Acute respiratory infections } \\
\text { of unspecified site }\end{array}$ & 3 & 8 & 5 \\
\hline UTI & $\begin{array}{l}\text { N39.0 Urinary tract infection, site not } \\
\text { specified }\end{array}$ & 3 & 6 & 3 \\
\hline \multirow[t]{2}{*}{$\begin{array}{l}\text { Vaginal issues (discharge, } \\
\text { irregular bleeding, etc) }\end{array}$} & $\begin{array}{l}\text { N89.9 Noninflammatory disorder } \\
\text { of vagina, unspecified }\end{array}$ & 3 & 3 & 0 \\
\hline & Totals & 674 & 805 & 131 \\
\hline \multicolumn{5}{|c|}{$\begin{array}{l}\text { ICD, International Classification of Diseases; COPD, chronic obstructive pulmonary disease; PTSD, posttraumatic stress disorder; STI, sexually transmitte } \\
\text { infection; UTI, urinary tract infection. }\end{array}$} \\
\hline \multicolumn{5}{|c|}{$\begin{array}{l}\text { aReports pulled before and after ICD system implementation. Table illustrates the number of ICD codes entered during project implementation. } \\
\text { bPreimplementation period spanned from September 6, } 2017 \text {, to March 22, } 2021 . \\
\text { 'Implementation period spanned from March 22, 2021, to June 15, 2021. }\end{array}$} \\
\hline
\end{tabular}

into the EHR. Additionally, the 20 most common diagnoses forms were posted on the walls at the provider stations along with pictures illustrating how to input the codes in the EHR. The more comprehensive map was attached to the nurse clipboards that accompanied each encounter. The first night the providers volunteered after receiving the email, the researcher would review with them how to input the diagnosis code and have them test the method on a practice patient, either in person or over the phone.
A starting report was pulled March 22, 2021, covering encounters between September 6, 2017, and March 22, 2021, for the 20 most common diagnoses. Another report was pulled at the completion of the implementation phase, on June 15, 2021, covering March 22, 2021 , to June 15,2021 . Willing providers and staff members were surveyed after implementation completion. The providers were asked whether they use the ICD codes, whether they would do so in the future, and whether they 
found it helpful when other providers had entered diagnoses. If they answered no to any of the questions, there were asked why, and whether they had any suggestions for improvements. The 4 staff members were asked whether they thought the data were helpful for their role and, if so, how they would use it.

\section{Surveys}

Surveys were conducted after the project was completed with willing and available providers and staff members in order to assess the utility of the project as well as to ensure future improvements and sustainability of the system.

\section{Provider surveys}

Do you currently input mapped $I C D-10$ codes when you chart for each encounter?

Yes No

If yes, do you intend to continue inputting the $I C D$ codes in your encounters in the future?

Yes No

If no to either question above, please explain:

Do you have any recommendations for making it easier to input ICD codes or another way to track patients' diagnoses?

\section{Staff surveys}

Is this data helpful for your role?

Yes No

If yes, how will you use this data?

\section{Results}

During the implementation phase, hypertension was the most common diagnosis seen at TOFC, accounting for 35 of 131 (27\%) top 20 diagnoses entered. Depression was second, accounting for about $20 \%$ of diagnoses. Posttraumatic stress disorder was the third most common, making up $18 \%$ of diagnoses. There were 157 encounters during the implementation phase and 128 $I C D$ diagnoses entered into the chart during this time period, suggesting that most encounters had a corresponding diagnosis code entered. See the Table for more details.

\section{Survey results \\ Provider surveys}

Six providers answered the survey questions. Four answered "yes" to both questions and 2 answered "no" to both questions. Reasons cited for why they did not input the $I C D$ codes included not remembering to enter the codes or not remembering how to enter the codes. Recommendations for making it easier included incorporating the diagnosis in the assessment section of the EHR instead of standing alone as its own section, replacing $/ C D-9$ codes with $/ C D-10$ codes on the maps, making more specific codes for options, like typing more mental health diagnoses, and implementing more training on how to enter the codes.

\section{Staff surveys}

Three of 4 staff members responded to the survey. All 3 indicated that the data collected from this project assisted in their role. Stated uses for this data included grant applications and funding; community education, such as presentations and outreach; program development and monitoring; quality improvement; supply purchasing (eg, medications in stock to treat most commonly seen conditions), scheduling clinics and providers; allocating resources and supplies; and accepting or rejecting medical supply donations.

\section{Discussion}

Before this project, 668 of the top 20 most common diagnosis codes were entered from when TOFC introduced use of the EHR in the clinic in 2017, until the beginning of the implementation phase of this project in March 2021. During the 3 months of the implementation phase, 131 diagnoses were entered, representing almost $20 \%$ of the amount that were entered in 3 and a half years. Pulling the reports for these 20 diagnoses took less than 1 hour. During the needs assessment phase of this project, diagnoses for 3 months were extracted from the EHR by combing through provider notes and extracting the data from the notes-a process that took 11 hours.

Knowledge of diagnoses and the reasons for clinic attendance help the clinic make decisions about staffing, resources, and services. The TOFC board of directors used this data to assist with the decision of whether or 
not to change the clinic's mission to include primary care as an official clinic function. The original purpose of the clinic was to address acute issues for people who lacked the resources for medical care. For example, a homeless person with an abscess could come to the clinic and have the abscess drained and treated. The results of this project illustrate that, in reality, most of the diagnoses actually seen in the clinic are more chronic in nature and require consistent, ongoing care. For instance, the project identified 52 clinic patients receiving consistent diabetic care. This type of data can help the clinic determine whether it should accept diabetes-associated donations and whether it needs to recruit a volunteer diabetes educator. Generally, this data can help guide other decisions as well, like what medications should be kept in the pharmacy, whether there are certain specialists the clinic should seek to partner with, and whether the clinic should embark on any particular education campaigns. By inputting ICD codes, diagnosis data are easily obtained to assist with future decisions.

A limitation of this project was that the reports could only be pulled within a certain time frame if the start date of the diagnosis was specified. As most providers did not indicate a start date with their entered diagnosis code, the only way to compare the before and after was to count the total before and the total after the implementation time frame. In other words, comparison reports could not be pulled retroactively, so some data on the less common diagnosis codes are missing from this paper, as reports for the comprehensive map were not pulled ahead of time. Providers may have omitted the start date when entering the diagnosis codes because many of these patients had their diagnoses for years-seeing different providers each time-so starting the diagnosis at that particular encounter did not make sense. Additionally, during training, although how to enter the start date was demonstrated, the emphasis and priority was placed on actually entering the $I C D$ code, in an effort to keep the process simple and increase participation.

\section{Conclusion}

Evidence-based care and informed decisionmaking require data. In a free clinic, this can be difficult to obtain due to limited staffing and the absence of billing and insurance requirements. $I C D$ codes and EHRs are powerful tools to collect data and information about clinic needs. This project improved TOFC's knowledge about what kind of patients and diagnoses they see.

Corresponding author: Sarah M. Shanahan, MSN, RN, Pacific Lutheran University School of Nursing, Ramstad, Room 214, Tacoma, WA 98447; slmarble94@gmail.com.

Financial disclosures: None.

doi:10.12788/jcom.0073

\section{References}

1. National Association of Free and Charitable Clinics. 2021 NAFC Member Data \& Standards Report. https://www.nafcclinics.org/ sites/default/files/NAFC\%202021\%20Data\%20Report\%20Final. pdf

2. Lee JS, Combs K, Pasarica M; KNIGHTS Research Group. Improving efficiency while improving patient care in a student-run free clinic. J Am Board Fam Med. 2017;30(4):513-519. doi:10.3122/jabfm.2017.04.170044

3. Lu KB, Thiel B, Atkins CA, et al. Satisfaction with healthcare received at an interprofessional student-run free clinic: invested in training the next generation of healthcare professionals. Cureus. 2018;10(3):e2282. doi:10.7759/cureus.2282

4. Tran T, Briones C, Gillet AS, et al. "Knowing" your population: who are we caring for at Tulane University School of Medicine's student-run free clinics? J Public Health (Oxf). 2020:1-7. doi:10.1007/s10389-020-01389-7

5. Sennett C. Healthcare reform: quality outcomes measurement and reporting. Am Health Drug Benefits. 2010;3(5): 350-352.

6. Mazzali C, Duca P. Use of administrative data in healthcare research. Intern Emerg Med. 2015;10(4):517-524. doi:10.1007/s11739-015-1213-9

7. Moons E, Khanna A, Akkasi A, Moens MF. A comparison of deep learning methods for ICD coding of clinical records. App/ Sci. 2020;10(15):5262. doi:10.3390/app10155262

8. Finkelman A. Quality Improvement: A Guide for Integration in Nursing. Jones \& Bartlett Learning; 2018. 